\title{
The Field Guide
}

If you are new at an organization that does not have a formal data privacy compliance program, you could start with Chapter 1, prepare a task list as you go, study or skip Chapter 2 on international data transfers (depending on how domestic or global your business is), and then work through Chapter 3 as you prepare documentation and execute your task list.

If you inherit or audit an existing program, start with the brief Chapter 4 first and then go through Chapters 1 to 3 .

If you just need a quick answer to a substantive question, check the Index at the end of the book and the summaries from $\mathrm{A}$ to $\mathrm{Z}$ in Chapter 5 for directions and perspective. The Field Guide cannot provide definitive answers to detailed questions, but it is intended to put phenomena into context and give you practical pointers and suggestions on how to solve problems, tackle tasks and find further information. 